\title{
Quantum anomalous Hall effect in graphene from Rashba and exchange effects
}

\author{
Zhenhua Qiao, ${ }^{1}$ Shengyuan A. Yang, ${ }^{1}$ Wanxiang Feng, ${ }^{2}$ Wang-Kong Tse, ${ }^{1}$ Jun Ding, ${ }^{2}$ Yugui Yao, $, 1, *$ \\ Jian Wang, ${ }^{3}$ and Qian Niu ${ }^{1}$ \\ ${ }^{1}$ Department of Physics, The University of Texas, Austin, Texas 78712, USA \\ ${ }^{2}$ Beijing National Laboratory for Condensed Matter Physics and Institute of Physics, Chinese Academy of Sciences, \\ Beijing 100190, China \\ ${ }^{3}$ Department of Physics and the Center of Theoretical and Computational Physics, The University of Hong Kong, \\ Hong Kong, China
}

(Received 10 May 2010; published 20 October 2010)

\begin{abstract}
We investigate the possibility of realizing quantum anomalous Hall effect in graphene. We show that a bulk energy gap can be opened in the presence of both Rashba spin-orbit coupling and an exchange field. We calculate the Berry curvature distribution and find a nonzero Chern number for the valence bands and demonstrate the existence of gapless edge states. Inspired by this finding, we also study, by first-principles method, a concrete example of graphene with Fe atoms adsorbed on top, obtaining the same result.
\end{abstract}

DOI: $10.1103 /$ PhysRevB.82.161414

PACS number(s): 73.43.-f, 72.25.Dc, 81.05.ue

Graphene is a fascinating material not only because of its extraordinary electrical, magnetic, and mechanical properties but also because of its Dirac-cone structure in its low-energy spectrum, which involves three binary internal degrees of freedom: spin, sublattice, and valley. ${ }^{1-4}$ Manipulating these degrees of freedom may turn out to be the most rewarding means of controlling the properties of graphene and related materials. For example, by breaking the $A B$ sublattice symmetry, an energy gap can be opened at the Dirac points, ${ }^{5-8}$ leading to the valley-contrasting Hall, magnetic and optical responses. ${ }^{9}$ The intrinsic spin-orbit coupling (SOC) is proposed to give rise to the quantum spin Hall effect, ${ }^{10,11}$ a quantized response of a transverse spin current to an electric field.

However, the intrinsic SOC in graphene is proved to be too weak to realize the quantum spin Hall effect under present experimental conditions. ${ }^{12,13}$ On the other hand, the extrinsic Rashba SOC from breaking the mirror symmetry of the graphene plane tends to destroy this effect. ${ }^{10}$ Rashba SOC can be very large for graphene grown on a substrate. ${ }^{14,15}$ For example, a $225 \mathrm{meV}$ of Rashba spin splitting has been observed for graphene grown on $\mathrm{Ni}^{15} \mathrm{We}$ expect that Rashba SOC should also be present for graphene on insulating substrate, or with dilute adsorbates. In the following, we show that although Rashba SOC is detrimental to the quantum spin Hall effect, it helps to realize another important topological phenomenon: the quantum anomalous Hall effect.

This effect is signaled by a quantized charge Hall conductance for an insulating state. Unlike the quantum spin Hall effect, the quantum anomalous Hall effect requires the breaking of time-reversal symmetry. Unlike the quantum Hall effect, which is induced by a strong magnetic field, the quantum anomalous Hall effect relies on the internal magnetization and SOC. The quantized value of the Hall conductance is related to a bulk topological number and is robust against disorder and other perturbations. ${ }^{16,17}$ Although there have been many theoretical studies of this unusual effect, ${ }^{18-23}$ no experimental observation has been reported so far.

In this Rapid Communication, we predict that the quan- tum anomalous Hall effect can be realized in graphene by introducing Rashba SOC and an exchange field. We also consider a concrete example with transition-metal atoms (e.g., Fe) adsorbed on top of graphene. The resulting broken structural symmetry gives rise to a Rashba-type SOC whereas the hybridization between the carbon $\pi$ state and the $3 d$-shell states of magnetic atoms produces a macroscopic exchange field. The Hall conductivity is found to be $\sigma_{y x}$ $=2 e^{2} / h$ when the Fermi level lies in the bulk gap. From first-principles calculations, we find that a bulk gap $\sim 5.5 \mathrm{meV}$ can be opened by adsorbing Fe atoms on top of graphene, which is readily accessible under current experimental conditions.

In the tight-binding approximation, the Hamiltonian for graphene in the presence of Rashba SOC and exchange field can be written as

$$
\begin{aligned}
H= & -t \sum_{\langle i j\rangle \alpha} c_{i \alpha}^{\dagger} c_{j \alpha}+i t_{\mathrm{SO}} \sum_{\langle i j\rangle \alpha \beta} \hat{\mathbf{e}}_{z} \cdot\left(\boldsymbol{\sigma}_{\boldsymbol{\alpha} \boldsymbol{\beta}} \times \mathbf{d}_{i j}\right) c_{i \alpha}^{\dagger} c_{j \beta} \\
& +\lambda \sum_{i \alpha} c_{i \alpha}^{\dagger} \sigma_{z} c_{i \alpha},
\end{aligned}
$$

where $c_{i}^{\dagger}\left(c_{i}\right)$ is the electron creation (annihilation) operator on site $i$, and $\boldsymbol{\sigma}$ are the Pauli matrices. The angular bracket in $\langle i, j\rangle$ stands for nearest-neighboring sites. The first term is the usual nearest-neighbor hopping term. The second term is the Rashba SOC with coupling strength $t_{\mathrm{SO}}$, and $\mathbf{d}_{i j}$ represents a unit vector pointing from site $j$ to site $i$. The third term corresponds to a uniform exchange field. ${ }^{24}$ Throughout this Rapid Communication, we measure the Fermi level, Rashba SOC, and exchange energy in units of the hopping parameter $t$.

By transforming the Hamiltonian of Eq. (1) into a $4 \times 4$ matrix $H(\boldsymbol{k})$ for each crystal momentum, the band structure of bulk graphene can be numerically obtained by diagonalizing $H(\boldsymbol{k})$. Figure 1 shows the evolution of the band structures with Rashba SOC $t_{\text {SO }}$ and exchange field $\lambda$. Panel (a) plots the band structure of the pristine graphene with Dirac cones centered at $\mathrm{K}$ and $\mathrm{K}^{\prime}$ points in the reciprocal space. Due to spin degeneracy, $\mathrm{K}$ and $\mathrm{K}^{\prime}$ points are fourfold degen- 


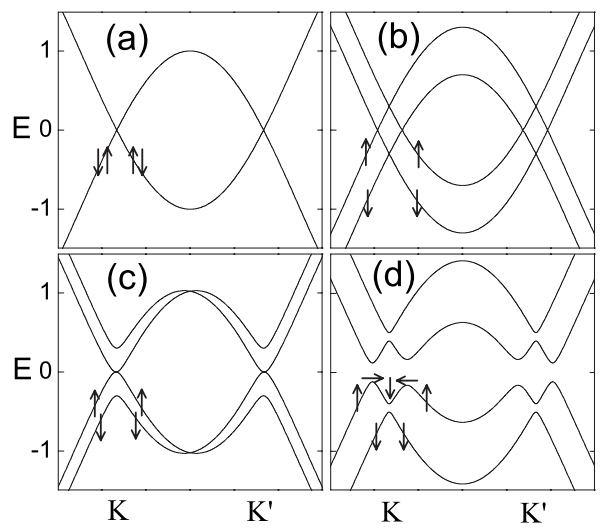

FIG. 1. Evolution of band structures of bulk graphene along the profile of $k_{y}=0$. Arrows represent the spin directions. (a) Pristine graphene: spin-up and spin-down states are degenerate; (b) when only exchange field $\lambda=0.4$ is applied, the spin-up/spin-down bands are upward/downward lifted with the four bands crossing near $\mathrm{K}$ and $\mathrm{K}^{\prime}$ points; (c) when only Rashba SOC $t_{\mathrm{SO}}=0.1$ is present, the spin-up and spin-down states are mixed around the band crossing points; (d) when both exchange field $\lambda=0.4$ and Rashba SOC $t_{\mathrm{SO}}$ $=0.1$ are present, a bulk gap is opened and all four bands become nondegenerate.

erate while other points are doubly degenerate. When only the exchange field is applied, the spin-up (spin-down) bands are pushed upward (downward) as shown in panel (b). When only Rashba SOC is turned on, spin-up and spin-down states are mixed around the band crossing points and spin degeneracy is lifted. A bulk gap is opened when both Rashba SOC and exchange field are present, and the four bands become completely nondegenerate. The presence of such a bulk gap indicates an insulating state. As we argue below, this insulating state is topologically nontrivial with gapless chiral edge states and exhibiting a quantized charge Hall conductance.

The gapless chiral edge states can be clearly seen from the energy spectrum of graphene ribbons. Figure 2(a) plots the band structure of graphene ribbons, with zigzag edges and 800 atoms across the width. The parameters used here are $t_{\mathrm{SO}}=0.2$ and $\lambda=0.18$. One can easily distinguish the gapless edge states from the bulk states. For a given Fermi level in the gap, there exists four different edge states labeled as $A$, $B, C$, and $D$. From $\boldsymbol{v}(\boldsymbol{k})=\frac{1}{\hbar} \frac{\partial E(\boldsymbol{k})}{\partial \boldsymbol{k}}$, one can find that states $A$ and $B(C$ and $D)$ propagate along the same $-x(+x)$ direction. Panel (b) plots the wave-function distributions $|\psi|^{2}$ of the four states across the width (only part of the ribbons with one zigzag edge are plotted). One can observe that the wave functions of the $A$ and $B$ states are localized at the left boundary, with noticeable values on about 30 carbon atoms whereas the wave functions of the $C$ and $D$ states are essentially zero in this region. The contrary occurs on the other boundary (not shown in the figure). This is topologically distinct from the helical edge states of the quantum spin Hall effect, where opposite spins propagate in opposite directions along the same boundary.

The emergence of chiral edge states in the bulk gap is intimately related to the topological property of the bulk Bloch states in the valence bands. This is characterized by the quantized charge Hall conductance: $\sigma_{y x}=\mathcal{C} e^{2} / h$, where

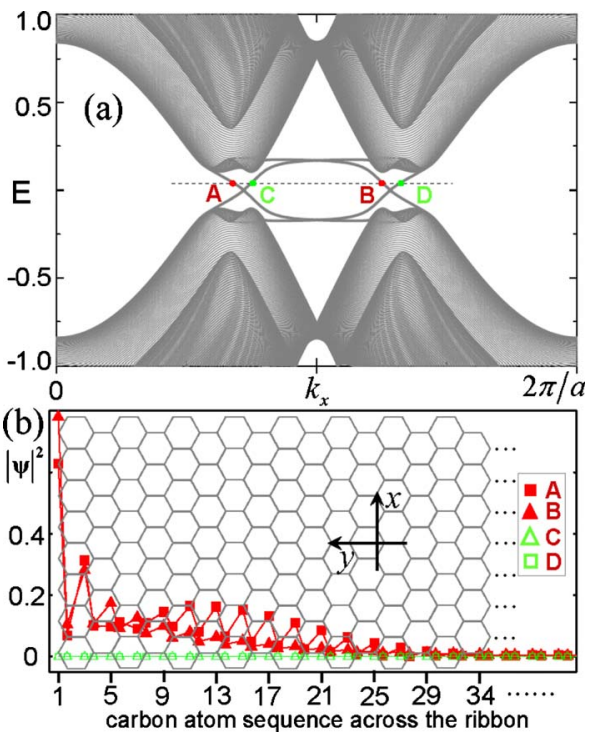

FIG. 2. (Color online) (a) Energy spectrum of zigzag-edged graphene ribbons with $t_{\mathrm{SO}}=0.2$ and $\lambda=0.18$. The Fermi level $E$ $=0.06$ corresponds to four different edge states $A, B, C$, and $D . a$ is the lattice constant. (b) Wave-function distributions $|\psi|^{2}$ across the width for the four edge states (only part of the ribbons are shown): $A, B$ states are localized at the left boundary, with noticeable values of $|\psi|^{2}$ on about 30 carbon atoms while $|\psi|^{2}$ of $C$ and $D$ states are completely zero in this region.

$\mathcal{C}$ is an integer known as the Chern number ${ }^{16,17}$ and can be calculated from

$$
\mathcal{C}=\frac{1}{2 \pi} \sum_{n} \int_{\mathrm{BZ}} d^{2} k \Omega_{n}
$$

where $\Omega_{n}$ is the momentum-space Berry curvature for the $n$th band $16,25,26$

$$
\Omega_{n}(\boldsymbol{k})=-\sum_{n^{\prime} \neq n} \frac{2 \operatorname{Im}\left\langle\psi_{n \boldsymbol{k}}\left|v_{x}\right| \psi_{n^{\prime} \boldsymbol{k}}\right\rangle\left\langle\psi_{n^{\prime} \boldsymbol{k}}\left|v_{y}\right| \psi_{n \boldsymbol{k}}\right\rangle}{\left(\omega_{n^{\prime}}-\omega_{n}\right)^{2}} .
$$

The summation is over all occupied bands below the bulk gap, $\omega_{n} \equiv E_{n} / \hbar$, and $v_{x(y)}$ is the velocity operator. The absolute value of $\mathcal{C}$ corresponds to the number of gapless chiral edge states along an edge of the two-dimensional system. ${ }^{27}$ In Fig. 3(a), we plot the Berry curvature distribution $\Omega$ for the valence bands in the momentum space. Panel (b) shows the profile of the Berry curvature along the $k_{y}=0$ intersection. We observe that the Berry curvature is peaked at the corners of the first Brillouin zone. It has the same sign at the inequivalent $\mathrm{K}$ and $\mathrm{K}^{\prime}$ points because the honeycomb lattice preserves the two-dimensional inversion symmetry for which $\Omega(\boldsymbol{k})=\Omega(-\boldsymbol{k})$. This is in contrast with the valley Hall effect, found in graphene system with $A B$ sublattice symmetry breaking, where the Berry curvature at $\mathrm{K}$ and $\mathrm{K}^{\prime}$ points has opposite signs. ${ }^{9}$ We obtain the Chern number from the curvature integration and find that $\mathcal{C}=2$, in agreement with the number of chiral edge states from tight-binding calculations. Therefore the charge Hall conductance is exactly quantized to $\sigma_{y x}=2 e^{2} / h$ when the Fermi level lies in the energy gap, i.e., the system realizes the quantum anomalous Hall effect. 

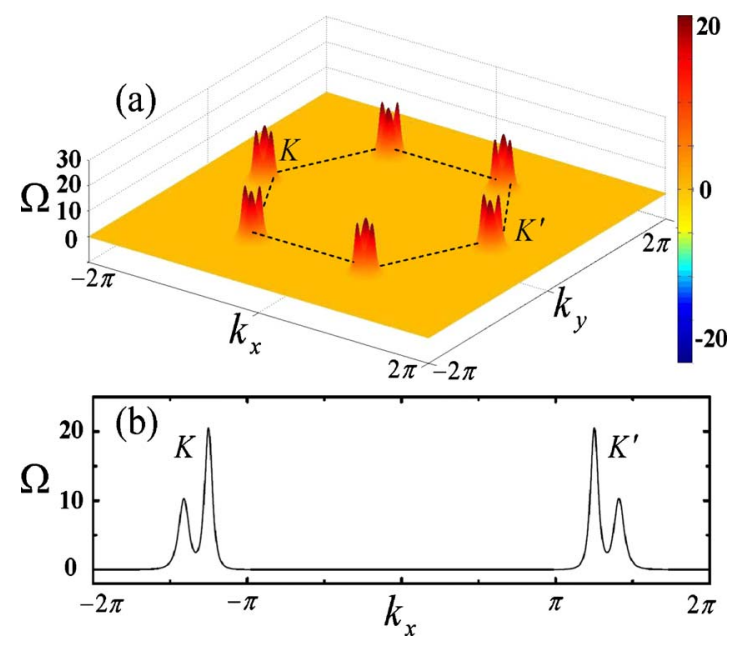

FIG. 3. (Color online) (a) Berry curvature distribution $\Omega$ (in units of $\left.e^{2} / h\right)$ of the valence bands in the momentum space. The first Brillouin zone is outlined by the dashed lines, and two inequivalent valleys are labeled as $\mathrm{K}$ and $\mathrm{K}^{\prime}$. (b) The profile of Berry curvature distribution along $k_{y}=0$. The parameters used here are $t_{\mathrm{SO}}=0.1$ and $\lambda=0.18$.

It is fundamentally different from the quantum Hall effect which relies on Landau-level quantization and quantum spin Hall effect which has vanishing charge Hall conductance. In the semiclassical picture, the quantum anomalous Hall effect can be viewed as a response to the Berry curvature in momentum space rather than the magnetic field in real space.

There is a simple explanation of the Chern number value $\mathcal{C}=2$. As shown in Fig. 1(d), there are two valence bands: the lower one is almost completely spin-down while the higher one involves a strong variation in the spin direction in the momentum space. By plotting the spin configuration in $k_{x}-k_{y}$ plane (see Fig. 4), we find that the spin textures around K and $\mathrm{K}^{\prime}$ form two separate Skyrmions of the same sign: ${ }^{28}$ spins point down in the central region (shown in red color) but point up in the outside region (shown in blue color). Since each Skyrmion contributes to a Chern number $\mathcal{C}=1$, Skyrmions at $\mathrm{K}$ and $\mathrm{K}^{\prime}$ points give rise to a total Chern number $\mathcal{C}=2$ in our model.

So far, we have predicted the occurrence of the quantum anomalous Hall effect for a model Hamiltonian with Rashba SOC and exchange field uniformly distributed on the honeycomb lattice. In the following, we consider another model in a similar spirit but with a nonuniform distribution: a
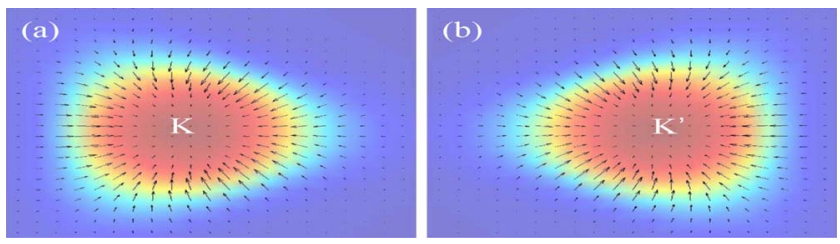

FIG. 4. (Color online) [(a) and (b)] Top view of the spin configurations of the higher valence band in the $k_{x}-k_{y}$ plane around $\mathrm{K}$ and $\mathrm{K}^{\prime}$ points. Blue/red (outer/inner) regimes represent the spin-up/ spin-down states. The arrows represent the spin direction at each point.

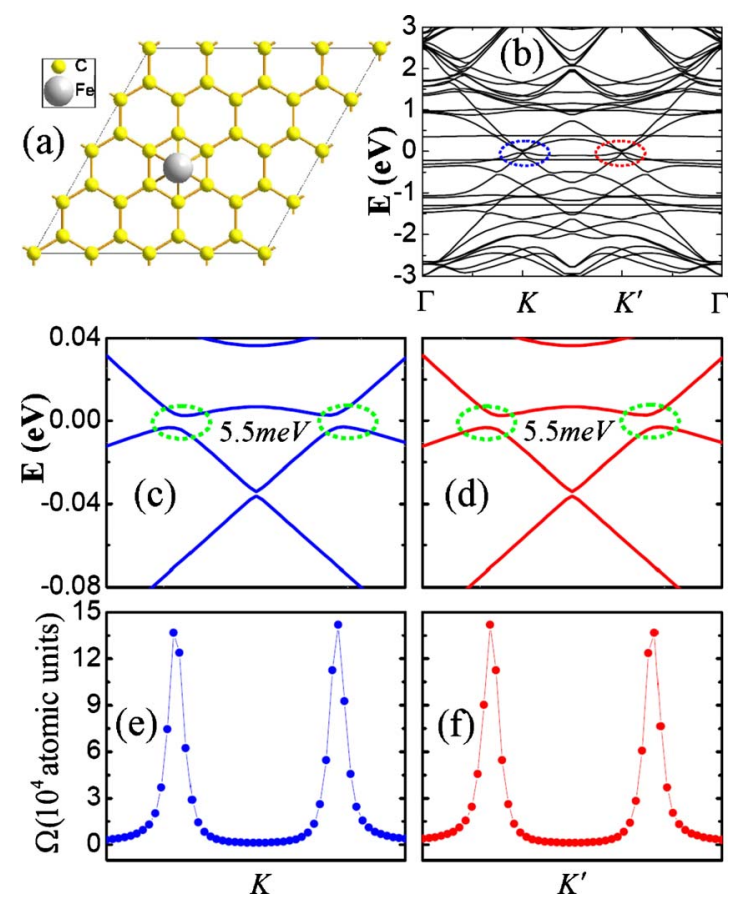

FIG. 5. (Color online) (a) Typical structure geometry of a Fe atom on top of the hollow position of a $4 \times 4$ supercell of graphene. (b) Bulk band structure with SOC included along the highsymmetry lines. The Fermi level is exactly located in the gap. [(c) and (d)] Zooming in of the band structure at $\mathrm{K}$ and $\mathrm{K}^{\prime}$ points. A gap $\sim 5.5 \mathrm{meV}$ circled by the green dotted curves is opened. [(e) and (f)] Berry curvature distribution $\Omega$ of the valence bands near $\mathrm{K}$ and $\mathrm{K}^{\prime}$ points. Note: the Berry curvature at $\mathrm{K}$ and $\mathrm{K}^{\prime}$ shares the same sign thus giving a nonzero Chern number $\mathcal{C}$.

graphene sheet with magnetized transition-metal atoms adsorbed on one side (i.e., top). Here we study a concrete case of Fe adsorbed graphene using first-principles method, and results with other kinds of adsorbates will be published in a separate paper. ${ }^{29}$ In our calculations, all atoms are allowed to relax along the normal direction of the graphene sheet but we have kept the experimental value of the lattice constant for graphene in the $x-y$ plane. Figure 5(a) shows a unit cell of a superlattice, with one $\mathrm{Fe}$ atom adsorbed on top of each 4 $\times 4$ supercell of the graphene sheet. The calculations were performed using the projected-augmented-wave method ${ }^{30}$ as implemented in the VASP package, ${ }^{31}$ and the generalized gradient approximation exchange-correlation potential ${ }^{32}$ was used.

We find that the most stable adsorption positions are on top of the hollow centers (with an equilibrium separation of $1.56 \AA$ ), which is consistent with the previous study without SOC. ${ }^{33,34}$ Figure 5(b) shows the bulk band structure with SOC of the iron taking into account. The magnetic moment of iron is found to be about $2 \mu_{B}$ in our calculations. This is consistent with the fact that there are totally eight electrons in the $3 d$ orbitals of the Fe atom after two $4 s$ electrons being transferred there. ${ }^{33}$ Therefore, no electron transfer occurs to the carbon atoms, thus the Fermi level is exactly located in the gap. Figures 5(c) and 5(d) zoom in the band structure around $\mathrm{K}$ and $\mathrm{K}^{\prime}$ points circled in panel (b). One can observe that a bulk gap around $5.5 \mathrm{meV}$ is opened at both $\mathrm{K}$ and $\mathrm{K}^{\prime}$ 
points. After obtaining the Bloch functions from the selfconsistent potentials, the corresponding Berry curvature $\Omega(\boldsymbol{k})$ near $\mathrm{K}$ and $\mathrm{K}^{\prime}$ points are calculated from the Kubo-formula: ${ }^{16,26} \Omega(\boldsymbol{k})=-\sum_{n} f_{n} \Omega_{n}(\boldsymbol{k})$, where $f_{n}$ is the equilibrium Fermi-Dirac distribution function. Figures 5(e) and 5 (f) plot the Berry curvature distribution $\Omega$ summing over all the valence bands. Two spikes near $K$ and $\mathrm{K}^{\prime}$ are clearly seen, and a Chern number of $\mathcal{C}=2$ for the valence bands is obtained by integrating over the whole Brillouin zone. This agrees with the result of our tight-binding model, despite the fact that the exchange and spin-orbit effects produced by the $\mathrm{Fe}$ atoms are clearly not uniform over the lattices.

In summary, we find that a nontrivial bulk gap in graphene can be produced in the presence of both Rashba SOC and exchange field, where we predict a quantum anomalous Hall conductance of $\sigma_{y x}$ quantized as $2 e^{2} / h .^{35}$ This is followed up with a more concrete example of graphene sheet with Fe atoms adsorbed on top. Our firstprinciples calculations show a bulk gap as large as $\sim 5.5 \mathrm{meV}$ can be opened at the Dirac points, producing the same topological effect. Recently, we became aware of a similar work $^{36}$ that demonstrates the quantum anomalous Hall effect in the bilayer graphene.

Z.Q. was supported by NSF (Grant No. DMR0906025) and Welch Foundation (Grant No. F-1255). Q.N. was supported by DOE (Grant No. DE-FG02-02ER45958, Division of Materials Science and Engineering) and Texas Advanced Research Program. Y.Y. was supported by NSF of China (Grants No. 10674163 and No. 10974231) and the MOST Project of China (Grants No. 2006CB921300 and 2007CB925000). J.W. was supported by RGC under Grant No. HKU7054/09P from the government of SAR of Hong Kong and LuXin Energy Group. The Texas Advanced Computing Center and Computer Center of the University of Hong Kong are gratefully acknowledged for computing assistance. *ygyao@aphy.iphy.ac.cn

${ }^{1}$ K. S. Novoselov et al., Science 306, 666 (2004); Nature (London) 438, 197 (2005).

${ }^{2}$ Y. Zhang et al., Nature (London) 438, 201 (2005).

${ }^{3}$ C. W. J. Beenakker, Rev. Mod. Phys. 80, 1337 (2008).

${ }^{4}$ A. H. Castro Neto et al., Rev. Mod. Phys. 81, 109 (2009), and references therein.

${ }^{5}$ S. Y. Zhou et al., Nature Mater. 6, 770 (2007).

${ }^{6}$ X. Peng et al., Nano Lett. 8, 4464 (2008).

${ }^{7}$ G. Giovannetti et al., Phys. Rev. B 76, 073103 (2007).

${ }^{8}$ W. Yao et al., Phys. Rev. Lett. 102, 096801 (2009).

${ }^{9}$ D. Xiao et al., Phys. Rev. Lett. 99, 236809 (2007).

${ }^{10}$ C. L. Kane et al., Phys. Rev. Lett. 95, 146802 (2005).

${ }^{11}$ C. L. Kane et al., Phys. Rev. Lett. 95, 226801 (2005).

${ }^{12}$ Y. G. Yao et al., Phys. Rev. B 75, 041401(R) (2007).

${ }^{13}$ H. Min et al., Phys. Rev. B 74, 165310 (2006).

${ }^{14}$ A. Varykhalov et al., Phys. Rev. Lett. 101, 157601 (2008).

${ }^{15}$ Y. S. Dedkov et al., Phys. Rev. Lett. 100, 107602 (2008).

${ }^{16}$ D. J. Thouless et al., Phys. Rev. Lett. 49, 405 (1982).

${ }^{17}$ M. Kohmoto, Ann. Phys. (N.Y.) 160, 343 (1985).

${ }^{18}$ F. D. M. Haldane, Phys. Rev. Lett. 61, 2015 (1988).

${ }^{19}$ M. Onoda et al., Phys. Rev. Lett. 90, 206601 (2003).

${ }^{20}$ C. X. Liu et al., Phys. Rev. Lett. 101, 146802 (2008).
${ }^{21}$ C. Wu, Phys. Rev. Lett. 101, 186807 (2008).

${ }^{22}$ S. Raghu et al., Phys. Rev. Lett. 104, 116401 (2010).

${ }^{23}$ R. Yu et al., Science 329, 61 (2010).

${ }^{24}$ O. V. Yazyev, Rep. Prog. Phys. 73, 056501 (2010), and references therein.

${ }^{25}$ M. C. Chang and Q. Niu, Phys. Rev. B 53, 7010 (1996).

${ }^{26}$ Y. G. Yao et al., Phys. Rev. Lett. 92, 037204 (2004).

${ }^{27}$ Y. Hatsugai, Phys. Rev. Lett. 71, 3697 (1993).

${ }^{28}$ T. H. R. Skyrme, Nucl. Phys. 31, 556 (1962); X. Z. Yu et al., Nature (London) 465, 901 (2010).

${ }^{29}$ Y. G. Yao et al. (unpublished).

${ }^{30}$ P. E. Blöchl, Phys. Rev. B 50, 17953 (1994).

${ }^{31}$ G. Kresse et al., Phys. Rev. B 54, 11169 (1996).

${ }^{32}$ J. P. Perdew et al., Phys. Rev. B 46, 6671 (1992).

${ }^{33}$ K. T. Chan et al., Phys. Rev. B 77, 235430 (2008).

${ }^{34}$ H. Johll et al., Phys. Rev. B 79, 245416 (2009).

${ }^{35} \mathrm{Z}$. H. Qiao et al., Quantum Anomalous Hall Effect in Graphene, Bulletin of the American Physical Society, 2010 March Meeting, http://absimage.aps.org/image/MWS_MAR102009-005805.pdf

${ }^{36}$ R. Nandkishore and L. S. Levitov, Phys. Rev. B 82, 115124 (2010). 